\title{
AN EQUIVALENCE THEOREM FOR EMBEDDINGS OF COMPACT ABSOLUTE NEIGHBORHOOD RETRACTS
}

\author{
J. L. BRYANT AND C. L. SEEBECK III ${ }^{1}$
}

In this paper we wish to prove the following theorem.

THEOREM 1. Suppose that each of $A_{0}$ and $A_{1}$ is a compact absolute neighborhood retract $(A N R)$ of dimension $k$ in Euclidean $n$-space $E^{n}$ $(2 k+2 \leqq n, n \geqq 5)$ such that $E^{n}-A_{i}$ is $1-U L C$ (uniformly locally simply connected) for $i=0,1$, and $f: A_{0} \rightarrow A_{1}$ is a homeomorphism such that $d(a, f(a))<\epsilon$ for each $a \in A_{0}$. Then there exists an $\epsilon$-push $h$ of $\left(E^{n}, A^{0}\right)$ such that $h \mid A_{0}=f$.

In [2] the authors showed that if $A$ is a $k$-dimensional polyhedron topologically embedded in $E^{n}(2 k+2 \leqq n, n \geqq 5)$ such that $E^{n}-A$ is $1=\mathrm{ULC}$, then for each $\epsilon>0$, there is an $\epsilon$-push $h$ of $\left(E^{n}, A\right)$ such that $h \mid A: A \rightarrow E^{n}$ is piecewise linear. Hence, a well-known theorem of Bing and Kister [1, Theorem 5.5] applies to prove Theorem 1 when $A_{0}$ is a polyhedron. In fact, Theorem 5.5 of [1], together with the techniques of Homma [4] and Gluck [3] and the following engulfing theorem proved in [2], make our result possible.

Theorem 2. Suppose that $A$ is a k-dimensional compact $A N R$ in $E^{n}(n-k \geqq 3, n \geqq 5)$ such that $E^{n}-A$ is $1-U L C$ and $\epsilon>0$. Then there exists $\delta>0$ such that if $f: A \rightarrow E^{n}$ is a $\delta$-homeomorphism and $U$ is an open subset of $E^{n}$ containing $f(A)$, then there exists an $\epsilon$-push $h$ of $\left(E^{n}, A\right)$ such that $h(U) \supset A$.

Following Gluck [3], we define an $\epsilon$-push $h$ of the pair $(X, A)$, where $X$ is a metric space and $A$ is a subset of $X$ such that $\bar{A}$ is compact, to be a homeomorphism of $X$ onto itself that is $\epsilon$-isotopic to the identity by an isotopy $h_{t}(t \in[0,1])$ of $X$ such that for each $t \in[0,1]$, $h_{t} \mid X-N_{\epsilon}(A)=$ identity. Other terminology used here is standard, and we shall assume that it is familiar to the reader.

Actually, the proof of Theorem 1 follows from known results, once we prove a

Lemma. Suppose that $A$ is a compact $A N R$ of dimension $k$ in $E^{n}$ $(2 k+2 \leqq n, n \geqq 5)$ such that $E^{n}-A$ is $1-U L C$ and $f: A \rightarrow E^{n}$ is an embedding such that $d(a, f(a))<\epsilon$ for each $a \in A$. Then for each $\delta>0$ there exists an $\epsilon$-push $h$ of $\left(E^{n}, A\right)$ such that $d(h(a), f(a))<\delta$ for each $a \in A$.

Received by the editors September 12, 1967. 5458 .

1 This research was supported in part by National Science Foundation Grant GP 
Theorem 1 is then proved exactly as is Theorem 4.4 of [3], and we shall not repeat the details of the constructions involved.

Proof of The Lemma. Given $\delta>0$, there exists $\eta>0$ such that if $a, b \in A$ with $d(a, b)<\eta$, then $d(f(a), f(b))<\frac{1}{2} \delta$. Let $N$ be a polyhedral neighborhood of $A$ in $E^{n}$ that retracts onto $A$ by a retraction $r: N \rightarrow A$ such that $d(x, r(x))<\frac{1}{2} \eta$ and $d(x, f r(x))<\epsilon$ for each $x \in N$. Let $T$ be a triangulation of $N$ with mesh less than $\frac{1}{4} \eta$ and let $T^{k}$ denote the $k$ skeleton of $T$, with $N^{k}=\left|T^{k}\right|$, the polyhedron of $T^{k}$.

The mapping $f r: N^{k} \rightarrow E^{n}$ has the property that if $x \in N^{k}, a \in A$, and $d(x, a)<\frac{1}{2} \eta$, then $d(f r(x), f(a))<\frac{1}{2} \delta$, since $d(r(x), a)<\eta$.

Let $g^{\prime}: N^{k} \rightarrow E^{n}$ be a piecewise linear embedding such that $d\left(g^{\prime}(x), f r(x)\right)<\frac{1}{2} \delta$ and $d\left(x, g^{\prime}(x)\right)<\epsilon$ for each $x \in N^{k}$. Since $2 k+2 \leqq n$, we may apply Theorem 5.5 of [1] to obtain an $\epsilon$-push $g$ of $\left(E^{n}, N^{k}\right)$ such that $g \mid N^{k}=g^{\prime}$. Notice that if $x \in N^{k}, a \in A$, and $d(x, a)<\frac{1}{2} \eta$, then $d(g(x), f(a)) \leqq d(g(x), f r(x))+d(f r(x), f(a))<\delta$. Thus there is an open set $U$ in $E^{n}$ containing $N^{k}$ such that the above implication is true for $x \in U$; that is, if $x \in U, a \in A$, and $d(x, a)<\frac{1}{2} \eta$, then $d(g(x), f(a))<\delta$. We need one additional fact concerning the open set $U$.

Sublemma. There exists $a \frac{1}{2} \eta$-push $\phi$ of $\left(E^{n}, A\right)$ such that $\phi(A) \subset U$.

Proof. Let $\tilde{T}^{n-k-1}$ be the dual $(n-k-1)$-skeleton of $T$ with $\tilde{N}^{n-k-1}=\left|\tilde{T}^{n-k-1}\right|$. Choose $\eta^{\prime}>0$ corresponding to $\frac{1}{4} \eta$ as in Theorem 2 . From the construction in the proof of Theorem V5 of [5], we can obtain an embedding $\psi$ of $A$ into $E^{n}$ such that $d(a, \psi(a))<\eta^{\prime}$ for each $a \in A$ and $\psi(A) \cap \tilde{N}^{n-k-1}=\varnothing$. Let $V$ be an open subset of $E^{n}$ containing $\psi(A)$ such that $V \cap \tilde{N}^{n-k-1}=\varnothing$.

By Theorem 2, there exists a $\frac{1}{4} \eta$-push $\phi_{1}$ of $\left(E^{n}, A\right)$ such that $\phi_{1}(V) \supset A$. Then $\phi_{1}^{-1}$ is a $\frac{1}{4} \eta$-push of $\left(E^{n}, A\right)$ and $\phi_{1}^{-1}(A) \cap \tilde{N}^{n-k-1}=\varnothing$. Since the mesh of $T$ is less than $\frac{1}{4} \eta$, the technique of Stallings [7] may be used to obtain a $\frac{1}{4} \eta$-push $\phi_{2}$ of $\left(E^{n}, \phi_{1}^{-1}(A)\right)$ such that $\phi_{2} \phi_{1}^{-1}(A) \subset U$. Then $\phi=\phi_{2} \phi_{1}^{-1}$ is the desired $\frac{1}{2} \eta$-push of $\left(E^{n}, A\right)$.

We complete the proof of the Lemma by setting $h=g \phi$. We may assume that $\eta$ is chosen sufficiently small so that the composition $g \phi$ is an $\epsilon$-push of $\left(E^{n}, A\right)$. Given $a \in A$, we have $d(\phi(a), a)<\frac{1}{2} \eta$ and $\phi(a) \in U$, so that $d(g \phi(A), f(a))=d(h(a), f(a))<\delta$.

The question as to whether Theorem 1 is true when $k=1$ and $n=4$ seems very hard to answer. The method used to prove Theorem 1 involves engulfing techniques that are valid only for $n \geqq 5$. It might be possible, however, to improve the codimension restriction by one if certain other conditions are satisfied. For example, Price has shown [6] that any two piecewise linear embeddings of a $k$-complex $K$ into $E^{n}(n=2 k+1)$ are equivalent by an isotopy of $E^{n}$ that is the identity outside a compact set if $H^{k}(K, Z)=0$. A natural question then is 
Question 1. Is Theorem 1 true with $n=2 k+1$ if $H^{k}\left(A_{0}, Z\right)=0$ ? In particular, one might consider a special case.

Question 2. Is Theorem 1 true with $n=2 k+1$ if $A_{0}$ is an absolute retract?

\section{REFERENCES}

1. R. H. Bing and J. M. Kister, Taming complexes in hyperplanes, Duke Math. J. 31 (1964), 491-511.

2. J. L. Bryant and C. L. Seebeck III, Locally nice embeddings of polyhedra, Quart. J. Math. Oxford Ser. (2) 19 (1968), 257-274.

3. H. Gluck, Embeddings in the trivial range, Ann. of Math. (2) 81 (1965), 195-210.

4. T. Homma, On the imbedding of polyhedra in manifolds, Yokohama Math. J. 10 (1962), 5-10.

5. W. Hurewicz and H. Wallman, Dimension theory, Princeton Univ. Press, Princeton, N. J., 1948.

6. T. M. Price, Equivalence of embeddings of $k$-complexes in $E^{n}$ for $n \leqq 2 k+1$, Michigan Math. J. 13 (1966), 65-69. 502.

7. J. Stallings, On topologically unknotted spheres, Ann. of Math. 77 (1963), 490-

Florida State University 